\title{
Tunable hybrid Tamm-microcavity states
}

\author{
Pavel S. Pankin ${ }^{1,2 *}$, Stepan Ya. Vetrov 2,3 , and IVan V. Timofeev 3,4 \\ ${ }^{1}$ FRC KSC SB RAS, Krasnoyarsk, 660036, Russia \\ ${ }^{2}$ Institute of Engineering Physics and Radio Electronics, Siberian Federal University, Krasnoyarsk 660041, Russia \\ ${ }^{3}$ Kirensky Institute of Physics, FRC KSC SB RAS, Krasnoyarsk, 660036, Russia \\ ${ }^{4}$ Institute of Nanotechnology, Spectroscopy and Quantum Chemistry, Siberian Federal University, Krasnoyarsk 660041, Russia \\ *Corresponding author: p.s.pankin@mail.ru
}

Compiled November 2, 2017

\begin{abstract}
Spectral manifestations of hybrid Tamm-microcavity modes in a 1D photonic crystal bounded with a silver layer and containing a nematic liquid crystal microcavity layer have been studied using numerical simulation. It is demonstrated that the hybrid modes can be effectively tuned owing to the high sensitivity of the liquid crystal to the temperature and external electric field variations. It is established that the effect of temperature on the transmission spectrum of the investigated structure is most pronounced at the point of the phase transition of the liquid crystal to the isotropic state, where the refractive index jump is observed.

(C) 2017 Optical Society of America

OCIS codes: (160.5298) Photonic crystals; (250.5403) Plasmonics; (140.3945) Microcavities; (230.3720) Liquid-crystal devices

http://dx.doi.org/10.1364/ao.XX.XXXXXX
\end{abstract}

\section{INTRODUCTION}

The optical Tamm state [1] is a surface state that is implemented when light is trapped between two mirrors. This state can occur at the interface between a photonic-crystal (PC) Bragg mirror and the other PC [2, 3], left-handed medium [4] or metallic mirror with negative permittivity $(\varepsilon<0)$ [5-7]. In the latter case, the light wave is merged with the surface plasmon, i.e., vibrations of free electrons at the surface of a metal and is called the Tamm plasmon polariton (TPP). In contrast to the surface plasmon polariton, the TPP can be excited for both TM and TE light polarizations even under normal incidence of light onto the interface. The TPPs have found application is lasers $[8,9]$, single-photon sources [10], sensors and optical switches [11, 12], optical filters [13], heat emitters [14, 15], and nonlinear amplifiers [16]. The TPP mode can be hybridized with the modes of other types simultaneously excited in a system, e.g., with the exciton mode [1,17-19] or surface plasmon polariton [20-22]. A new type of waveguide modes arises upon hybridization of two TPPs localized at the edges of a PC bounded with metallic or nanocomposite layers [7, 23, 24].

In recent years, close attention of researchers has been focused on the hybrid modes arising during simultaneous excitation of the TPP and microcavity (MC) mode in a PC system [25]. In studies [26, 27], the hybrid Tamm states were experimentally found. The resonance wavelengths were tuned by changing the polarization of incident light and by designing a structure with the embedded metallic layer of variable thickness and its scanning by a small-aperture light beam. The authors of $[28,29]$ proposed a new design of CuPc-PTCBI-based organic solar cells, where they implemented an idea of dual-states-induced broaderband absorption corresponding to the hybrid modes. The idea of emitting light at two resonance wavelengths corresponding to the hybrid modes underlies the design of white top-emitting organic light-emitting devices (WOLEDs) based on the twocomplementary-color strategy [30]. The experimental WOLEDs have the improved viewing characteristics and electroluminescence efficiency at the high quality of white color maintained. The WOLEDs have a great potential of application in energyefficient solid-state lighting sources and full-color flat-panel displays. In addition, the formation of three hybrid modes upon simultaneous excitation of a pair of Tamm modes and one microcavity mode was investigated. One of the hybrid modes ensures the extraordinary field amplification in a MC, which opens the way to intensification of the nonlinear optical effects [31]. The hybridization makes it possible not only to enhance the field inside a MC, but also to weaken absorption in the metallic layers embedded in the structure, which is important for designing vertical cavity lasers [32]. In the above-cited works, the resonance wavelengths and light energy distribution over a structure could be tuned by selecting parameters of the structure during its fabrication only.

The authors of $[33,34]$ proposed to electrically control the Tamm state occurring at the interface between two PCs, one of 
which contains a nematic liquid crystal (nematic). This can be realized owing to the high sensitivity of orientation in nematic layers to the applied voltage, which is reflected on the refractive indices of the layers and shifts the PC band gap and thereby the Tamm state wavelength.

The tuning of a bare TPP mode was accomplished by changing the thickness of the first PC layer adjacent to the metal [19, 26], the polarization of the incident light [7], the metal layer material [35] or the appropriate tuning of PC bandgap position [5].

The possibility of controlling MC modes in a 1D PC containing the nematic as a defect was demonstrated in [36-38] The MC modes were controlled using temperature, electric, and magnetic fields applied to the defect layer and by changing the angle of incidence and polarization of light.

In this work, we propose to tune the hybrid Tamm MC states via controlling the MC mode involved in the hybridization. The MC mode is controlled via affecting the nematic defect in a 1D $\mathrm{PC}$, which plays the role of a MC, by temperature or electric fields. The PC is coated with a silver layer, which allows exciting the TPP at the PC/metal interface simultaneously with the MC mode on a defect.

\section{MODEL}

The model under study is a 1D PC containing the nematic defect bounded with a silver film [Fig. 1]. The PC unit cell consists of zirconium dioxide $\mathrm{ZrO}_{2}$ and silicon dioxide $\mathrm{SiO}_{2}$ with respective refractive indices and thicknesses of $n_{a}=2.04, W_{a}=52 \mathrm{~nm}$ and $n_{b}=1.45, W_{b}=102 \mathrm{~nm}$. The defect layer with a thickness of $L=2.13 \mu \mathrm{m}$ is filled with a planar oriented 4-n-pentyl-4cyanobiphenyl (5CB) nematic. The nematic director, i.e., the unit vector of the preferred orientation of molecules, is aligned along the $x$ axis of the system. The $5 \mathrm{CB}$ nematic undergoes a sequence of phase transitions: crystal $-22.5^{\circ} \mathrm{C}-$ nematic $-35^{\circ} \mathrm{C}$ - isotropic liquid. The PC is coated with a silver film with a thickness of $W_{A g}=50 \mathrm{~nm}$ on its one side; its permittivity is determined using the Drude-Sommerfeld approximation

$$
\varepsilon_{A g}(\omega)=\varepsilon_{\infty}-\frac{\omega_{p}^{2}}{\omega(\omega+i \gamma)}
$$

Here, $\varepsilon_{\infty}$ is the constant, which takes into account the contributions of interband transitions of bound electrons; $\omega_{p}$ is the plasma frequency; and $\gamma$ is the reciprocal electron relaxation time. For silver, we have $\varepsilon_{\infty}=5, \omega_{p}=9 \mathrm{eV}$, and $\gamma=0,02 \mathrm{eV}$ [39]. The total number of layers, including nematic and silver, is $\mathrm{N}=$ 24.

The transmission spectra of the structure and light field energy distribution in it were found numerically using the transfer matrix method [40] at the normal incidence of light. We investigated the incidence of waves polarized along the $x$ and $y$ axes.

The LC structure in the applied voltage was calculated using the free energy variation technique [41]. The elastic energy of the LC layer is

$$
2 F_{k}=\left(k_{11} \cos ^{2} \theta+k_{33} \sin ^{2} \theta\right)(d \theta / d z)^{2} .
$$

Here, $\theta$ is the tilt angle of the LC director relative to the $x$ axis and coefficients of elasticity $k_{11}$ and $k_{33}$ correspond to the longitudinal bend and splay, respectively. The electrostatic energy of the LC layer is expressed as

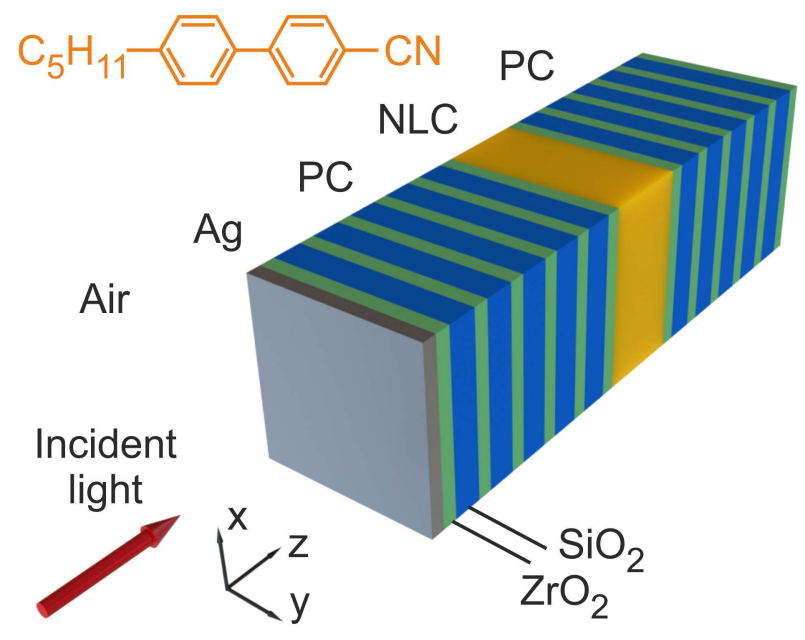

Fig. 1. Photonic crystal with a nematic defect bounded with a silver film. Inset: structural chemical formula of the $5 \mathrm{CB}$ nematic.

$$
2 F_{e}=-\vec{D} \vec{E}=-D_{z}^{2} / \varepsilon_{0}\left(\varepsilon_{\perp} \cos ^{2} \theta+\varepsilon_{\|} \sin ^{2} \theta\right)
$$

Here, $\vec{E}$ is the vector of electric field applied to the LC layer, $\vec{D}$ is the vector of electric induction in the bulk of the $L C, \varepsilon_{\perp}$ and $\varepsilon_{\|}$ are the LC permittivities transverse and longitudinal relative to the director, and $\varepsilon_{0}$ is the permittivity of free space.

The free energy variation $F=F_{k}+F_{e}$ can be described as

$$
\delta F=\frac{\delta F_{k}}{\delta \theta} \delta \theta+\frac{\delta F_{e}}{\delta \theta} \delta \theta .
$$

Here, $\delta / \delta \theta$ is the operator of variational derivative with respect to orientation. In the equilibrium configuration, free energy variation (4) should be zero, regardless of the $\delta \theta$ value. Taking into account formulas $(2,3)$, we arrive at the equation for the angle $\theta(z)$

$$
\begin{aligned}
& \left(k_{11} \cos ^{2} \theta+k_{33} \sin ^{2} \theta\right) \frac{d^{2} \theta}{d z^{2}}+ \\
& \frac{\left(k_{33}-k_{11}\right) \sin (2 \theta)}{2}\left(\frac{d \theta}{d z}\right)^{2}+ \\
& \frac{D_{z}^{2}\left(\varepsilon_{\|}-\varepsilon_{\perp}\right) \sin (2 \theta)}{2 \varepsilon_{0}\left(\varepsilon_{\perp} \cos ^{2} \theta+\varepsilon_{\| \mid} \sin ^{2} \theta\right)^{2}}=0 .
\end{aligned}
$$

If the problem is one-dimensional and there is no divergence of induction $(\nabla \vec{D}=0)$, we may conclude that this quantity is constant over the entire LC volume $\left(|\vec{D}|=D_{z}=\right.$ const $\left.(z)\right)$ and can be related to voltage $U$ applied to the defect layer as

$$
U=\int_{0}^{L} E_{z} d z=\frac{D_{z}}{\varepsilon_{0}} \int_{0}^{L} \frac{d z}{\varepsilon_{\perp} \cos ^{2} \theta+\varepsilon_{\|} \sin ^{2} \theta} .
$$

The function of distribution of the tilt angle $\theta(z)$ is found from the joint solution of Eqs. $(5,6)$ with regard to the boundary conditions, which are determined by the rubbing direction on the PC mirrors. 


\section{RESULTS AND DISCUSSION}

\section{A. Hybrid modes}

The formation of hybrid modes was demonstrated at the normal incidence of the $x$-polarized light wave, when the nematic director is parallel to the electric field. The mode coupling phenomenon was investigated assuming the 5CB extraordinary and ordinary refractive indices $n_{e}=1.71+0.00039 i$ and $n_{o}=1.54+0.00039 i$ [42] with disregard of dispersion at a temperature of $25^{\circ} \mathrm{C}$. The estimated imaginary part of the nematic refractive index was take from experimental work [43]. This quantity determines the total nematic extinction, with contribution of light absorbtion and scattering.

If we eliminate the silver layer, the structure will turn to the MC filled with the nematic bound by Bragg mirrors. In the spectrum shown in Fig. 2(a), one can see several transmission peaks localized in the PC band gap. These peaks correspond to bare $\mathrm{MC}$ modes. If there is no nematic layer in the structure, then the latter represents two conjugate mirrors: metallic and Bragg. At the interface between them, a bare TPP is maintained. The TPP manifests itself in the spectrum as a narrow peak [Fig. 2(a)]. The parameters of the structure are found to equate the wavelengths of the TPP and a MC mode. If the PC contains both the nematic and silver film, then both modes are simultaneously excited. This leads to their coupling and formation of two hybrid TammMC modes, short- and long-wavelength. This can be seen in the spectrum as splitting of the transmission peak [Fig. 2(b)].

The splitting value characterizes the mode coupling and, in the investigated case, is equal to $12.8 \mathrm{~nm}$. The splitting value can be increased by enhancing the spatial overlap of the coupled modes. This can be achieved by decreasing a number of PC layers between the silver film and nematic defect. The field energy is distributed between coupled modes localized both at the PC/metal interface and in the bulk of the MC [Fig. 2(c,d)].

\section{B. Temperature control of the hybrid modes}

To calculate temperature tuning of the spectra, we used the experimental values of the temperature dependences of refractive indices $n_{e}$ and $n_{0}$ [44]. Figure 3 shows the calculated temperature transmission spectra of the structure in the wavelength range corresponding to the hybrid modes.

In the figure, one can see the motion of peaks corresponding to the hybrid modes. Since the bare TPP wavelength remains invariable, the motion of peaks can be explained by the sensitivity of the MC mode wavelengths to the applied temperature field [36]. As the temperature increases, the refractive index $n_{e}$ decreases, which leads to a decrease in the MC optical thickness and, consequently, to the blue shift of MC modes [Fig. 3(a)]. It follows from the Fabry-Perot resonance condition that [45]:

$$
m \lambda_{m}=2 L n
$$

Here, $m$ is the number of MC mode, $\lambda_{m}$ is its wavelength, and $n$ is the refractive index of the defect layer. Actually the MC mode standing wave antinodes are shifted beyond the defect layer thickness $L$. The corresponding wavelength shift is neglected in Eq. (7) .

The wavelength shift of MC modes leads to the shift of hybrid Tamm-MC modes. In the investigated temperature range, the shift of the long-wavelength mode attains $7 \mathrm{~nm}$ and the shift of the short-wavelength mode, $12 \mathrm{~nm}$. At the point of the phase transition between nematic and isotropic liquid at $t_{0}=35.0^{\circ} \mathrm{C}$, the jump of refractive index $n_{e}$ is observed [46]. This leads to
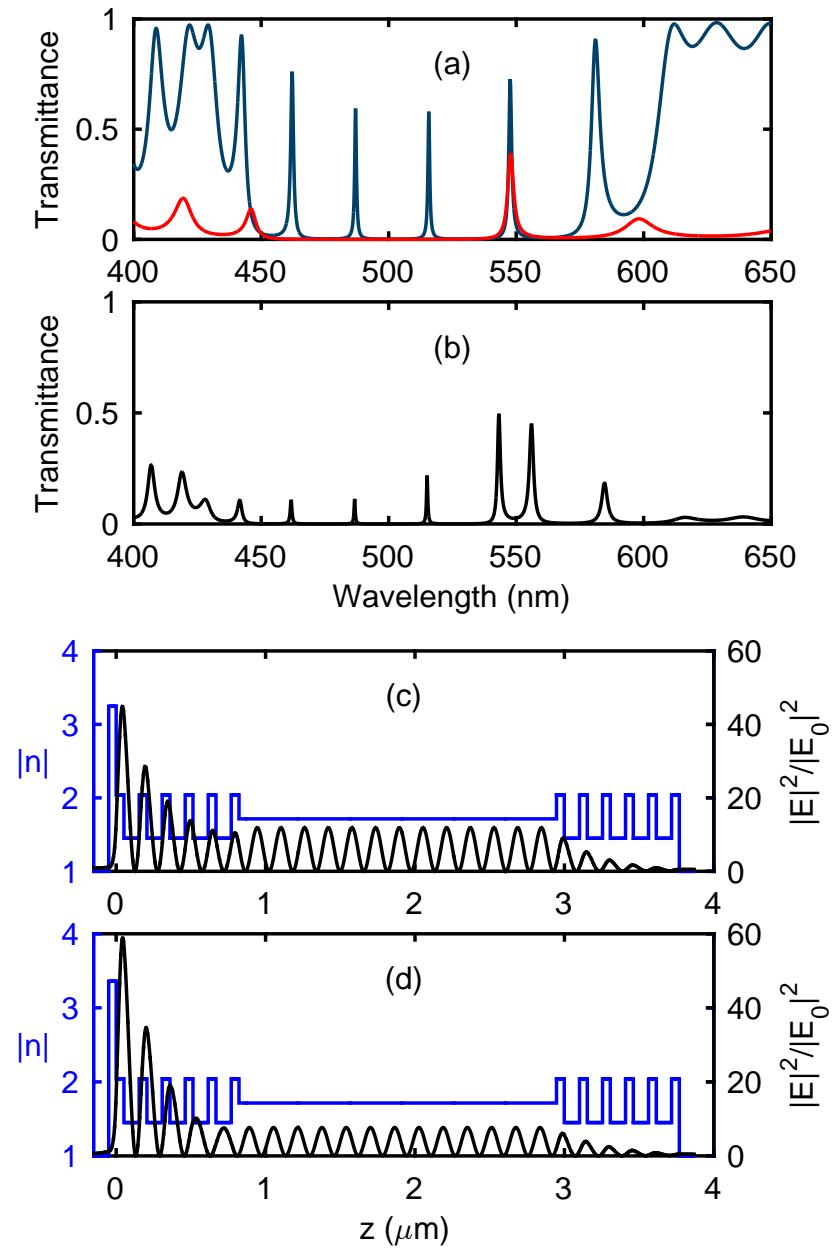

Fig. 2. (a) Transmission spectra of the PCs without metallic layer (blue) (bare MC mode at $547.7 \mathrm{~nm}$ ) and without nematic defect (red) (bare TPP at $547.7 \mathrm{~nm}$ ). (b) Transmission spectrum of the investigated structure (hybrid Tamm-MC modes at 543.2 and $556 \mathrm{~nm})$. The nematic director is parallel to the electric field ( $x$ polarization). Spatial distribution of the refractive index and electric field energy of the light wave in the hybrid Tamm-MC modes at wavelengths of (c) 543.2 and (d) $556 \mathrm{~nm}$. 

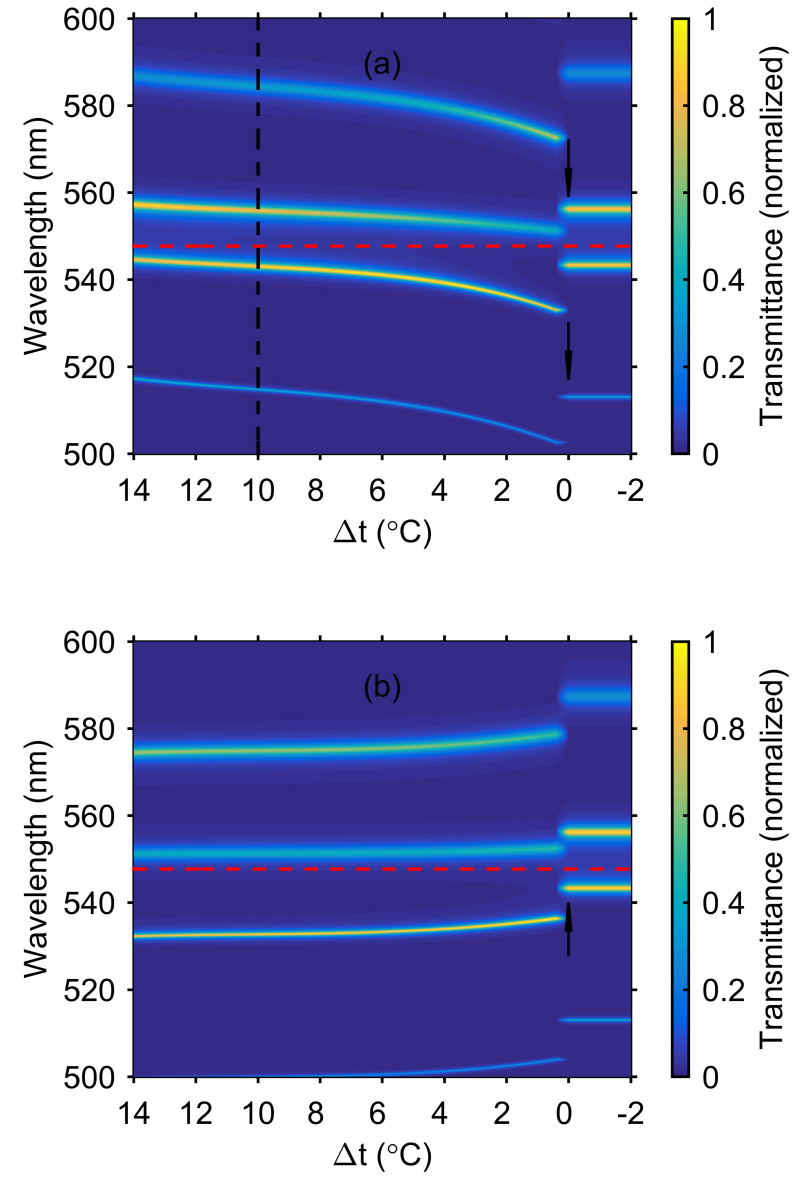

Fig. 3. Normalized transmission spectrum $T / T_{\max }$ of the structure vs temperature detuning $\Delta t=t_{0}-t$, where $t_{0}=35.0^{\circ} \mathrm{C}$ is the phase transition temperature. (a) The nematic director is parallel to the electric field ( $x$ polarization); $T_{\max }=0.55$. The black line corresponds to the spectrum in Fig. 2(b). (b) The nematic director is perpendicular to the electric field vector ( $y$ polarization); $T_{\max }=0.52$. Arrows show the direction of the jump of MC modes involved in the formation of the hybrid state. The red line corresponds to the bare TPP wavelength. the stepwise blue shift of the MC modes, which attains $20 \mathrm{~nm}$. During the phase transition, the MC mode coupled with the TPP turns to the bare MC mode. At the same time, the neighboring MC mode jumps toward the TPP; as a result, they are coupled and new bound Tamm-MC states are formed. With a further increase in temperature, the refractive index of the isotropic liquid remains almost invariable; therefore, the hybrid mode wavelengths after the phase transition do not change.

The temperature behavior of refractive index $n_{0}$ [Fig. 3(b)] is opposite to [Fig. 3(a)]. The resonant wavelength grows with temperature and the hybrid modes undergo the red shift. Through $14^{\circ}$ temperature interval the long-wavelength hybrid mode shifts by $2 \mathrm{~nm}$ and the short-wavelength mode shifts by $5 \mathrm{~nm}$. At the phase transition point, a stepwise red shift of the MC modes of up to $10 \mathrm{~nm}$ is observed. This leads to the jump of hybrid modes. Comparison of Figs. 3(a) and 3(b) allows us to conclude that the temperature spectra are extremely sensitive to the polarization direction of the incident light.

\section{Electric-field control of the hybrid modes}

The electric field applied to the LC defect layer leads to the orientational transition. This effect is called Fredericks transition [46]. Figure 4(a) shows the calculated transmission spectrum of the structure for the $x$-polarized light in the variable voltage applied to the defect layer. It can be seen that at $U<U_{c}=0.74$ $\mathrm{V}$ the spectrum does not change. This is caused by the threshold character of the Fredericks transition. In this voltage range, the spectrum corresponds to Fig. 2(b).

At the voltages above critical voltage $U_{c}$, the blue shift of hybrid modes is observed. This can be explained as follows. The LC in the defect layer has the initial planar orientation; the electric voltage is applied perpendicular to the defect layer. Since the permittivity along the director is larger than the transverse component $\left(\varepsilon_{\|}>\varepsilon_{\perp}\right)$, we observe the so-called Fredericks Stransition [46]. LC director begins to deviate from the $x$ axis and to align parallel to the applied field, i.e., along the $z$ axis.

The distribution of the tilt angle $\theta(z)$ as a function of applied voltage $U$ was found by numerical solution of Eqs. $(5,6)$ using the gradient descent technique $[43,47]$. The boundary conditions correspond to the initial planar LC orientation $\theta(0)=\theta(L)=0$. The calculation was performed for the following $5 \mathrm{CB}$ parameters at a temperature of $25^{\circ} \mathrm{C}: k_{11}=5.9 \mathrm{pN}, k_{33}=9.9 \mathrm{pN}, \varepsilon_{\|}=18$, and $\varepsilon_{\perp}=6$ [48]. Taking into account the tilt of LC director, the LC refractive index for the $x$-polarized radiation is [45]

$$
n(z)=\frac{n_{e} n_{o}}{\sqrt{n_{e}^{2} \sin ^{2} \theta(z)+n_{o}^{2} \cos ^{2} \theta(z)}} .
$$

It can be seen that the refractive index of the defect LC layer decreases with increasing tilt angle. This is shown by comparison of the refractive index distributions over the structure in Figs.4 and 2. One can see that under the action of applied voltage, the refractive index of the nematic layer decreases over the entire LC volume and is minimum at the center of the MC where there are no boundary effects on PC mirrors. With regard to Eq.(7), this leads to the blue shift of the MC modes and, simultaneously, hybrid Tamm-MC modes. In this case, at $U>4.5 \mathrm{~V}$, the wavelengths of hybrid modes almost stop changing. This is due to the fact that the LC director approaches the homeotropic state and its rotation slows down. Except for a thin surface regions, the LC director aligns along the $z$ axis. Then, the refractive index for the $x$-polarized light becomes equal to the ordinary refractive index $n_{0}$. This can be seen also from Eq.(8), if we take $\theta(z)=90^{\circ}$. 

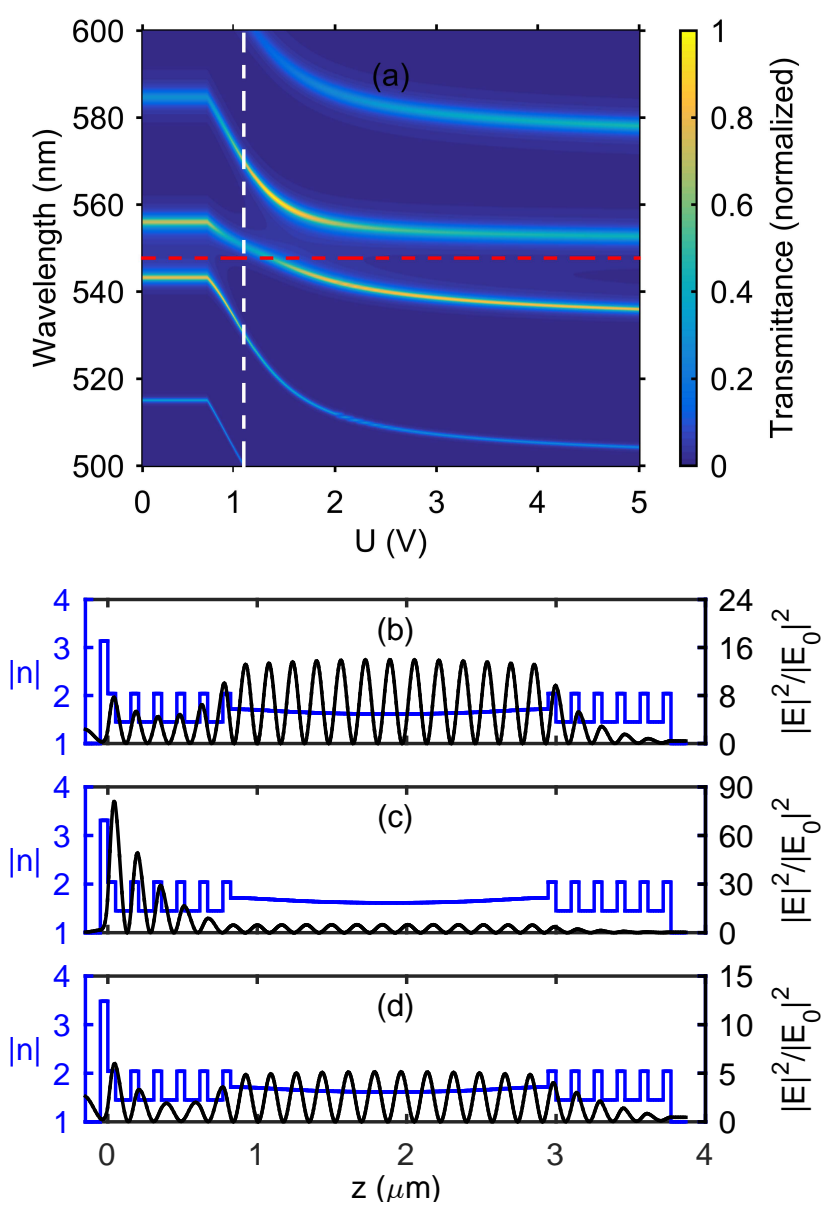

Fig. 4. (a) Normalized transmission spectrum $T / T_{\max }$ of the structure vs applied voltage for the $x$-polarized light; $T_{\max }=0.6240$. The external field is directed along the $z$ axis. The red line corresponds to the wavelength of the bare TPP. Spatial distribution of the refractive index and electric field energy of the light wave in hybrid Tamm-MC modes at $U=1.1$ $\mathrm{V}$ (white line in Fig. 4(a)) at wavelengths of (b) 530.5, (c) 550.5, and (d) $570 \mathrm{~nm}$.
It can be seen from Fig. 4(a) that at a voltage of about $U=1.4$ $\mathrm{V}$, the hybrid mode crosses the bare TPP wavelength. Such a behavior of the hybrid mode contradicts the well-known general theory for two modes coupling, when their wavelengths shift in opposite directions and avoid to cross each other $[49,50]$. In our case the behavior can be explained by the triple hybridization. As the voltage increases, one MC mode initially coupled with the TPP moves away from it and turns to the bare MC mode at high voltages. At the same time, the neighboring MC mode approaches the TPP and is hybridized with it. In the intermediate region, where both $\mathrm{MC}$ modes are sufficiently close to the TPP, three modes are hybridized. Such a behavior of the spectral peaks was observed in [31], where the hybrid states formed by two TPPs and one MC mode were investigated.

This assumption of triple hybridization is confirmed by the light field energy distribution over the structure at a voltage of $U=1.1 \mathrm{~V}$ [see Figs. 4(b,c,d)]. It can be seen that the light field in each of the three modes has localization maxima both in the bulk of the $\mathrm{MC}$ and at the $\mathrm{PC} /$ metal interface. The mode close to the bare TPP in wavelength [Fig. 4(c)] is mainly localized at the $\mathrm{PC} /$ metal interface, whereas the other two modes are more like the bare TPP modes and are mainly localized in the bulk of the MC [Fig. 4(b,d)]. Comparison of Figs. 4 and 2 shows that we can tune not only the wavelength of hybrid modes, but also the light energy localization at the PC/metal interface, which is important for the TPP applications. The transmission spectra of the $y$-polarized wave do not change, since in this case the refractive index of the LC remains equal to the ordinary refractive index $n_{0}$ during reorientation of director in the $x z$ plane.

In addition, note several technical points. In the experiment the voltage can be applied through indium tin oxide (ITO) layers embedded in the structure on each side from the nematic [38]. In this case, the MC mode acquires an additional phase delay and is partially absorbed in the ITO layers. The calculation shows that at an ITO layer thickness of $30 \mathrm{~nm}$, the transmittance in the peaks changes by several percent and the peaks undergo a red shift of several nanometers. WOLEDs technology based on TPP-MC modes requires two wide peaks in visible spectrum range. For this goal one may decrease the thickness of nematic layer increasing the distance between the peaks. Additionally, decreasing the number of PC layers leads to peak broadening. Also liquid crystal mixtures with a wide nematic range $\left(-40^{\circ} \mathrm{C}\right.$ to $+100^{\circ} \mathrm{C}$ ) [51] may be used for applications. The response time to the applied electric field is less than $10 \mathrm{~ms}$, estimated for conventional nematic liquid crystal cells [46].

\section{Tuning the hybrid mode wavelengths}

Upon variation in thickness $d$ of the first $\mathrm{ZrO}_{2}$ layer adjacent to the silver layer, the phase of reflectance from the PC changes. Since the condition of phase matching between the reflectances from the metallic and Bragg mirrors should be satisfied, the TPP wavelength changes [5]. This makes it possible to tune the hybrid mode wavelengths [Fig. 5].

The wavelengths of the MC modes are almost insensitive to the growth of thickness $d$, while the bare TPP mode undergoes a red shift and pass through the entire PC band gap. In this case, the sequential hybridization with all the MC modes occurs. The similar situation is observed when the electric field vector is perpendicular to the nematic director ( $y$ polarization).

The significant dependence of the transmission peak wavelengths on the first layer thickness makes it possible to design a tunable filter on the basis of the proposed structure. For this 


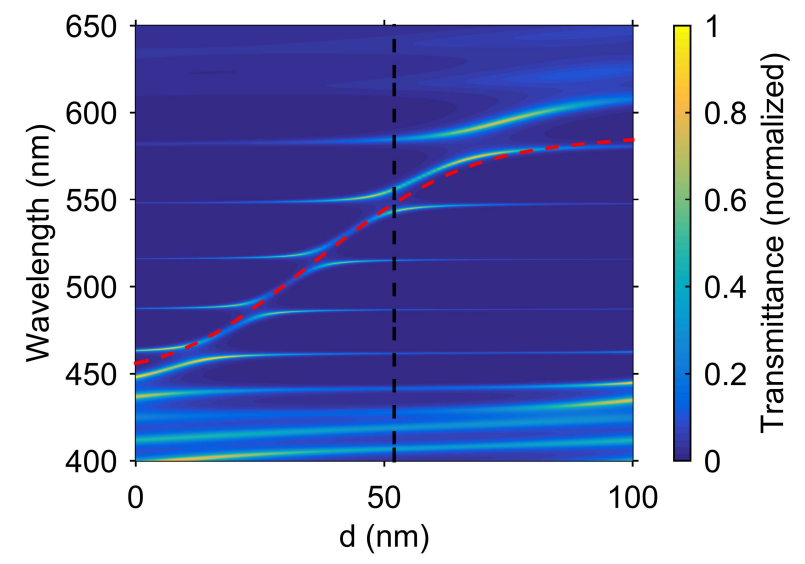

Fig. 5. Normalized transmission spectrum $T / T_{\max }$ of the structure vs thickness $d$ of the first $\mathrm{ZrO}_{2}$ layer. The nematic director is parallel to the electric field ( $x$ polarization); $T_{\max }=0.7186$. The black line corresponds to the spectrum in Fig. 2(b). The red line corresponds to the bare TPP wavelength.

purpose, the first layer should have a variable thickness, e.g., be wedge-like $[19,26]$.

\section{CONCLUSIONS}

Transmission spectra of a 1D PC coated with a thin silver layer were calculated. The photonic crystal under study contains the defect layer infiltrated with 5 CB nematic. The existence of hybrid modes formed by the Tamm plasmon polariton and microcavity mode in the structure was demonstrated. It was shown that the light field energy is distributed over the coupled modes.

The possibility of temperature tuning of the spectra was demonstrated, which is caused by the sensitivity of the nematic refractive index to the applied temperature field. The existence of the hybrid mode jump at the point of the phase transition nematic-isotropic liquid was demonstrated. The polarization sensitivity of the spectra was demonstrated.

The possibility of tuning the spectra by the electric field was demonstrated, which is caused by the sensitivity of the nematic refractive index to the applied electric field. It was shown that at the shift of the microcavity modes under the action of a field, the Tamm mode is sequentially hybridized with the neighboring microcavity modes. It was shown that there is the voltage range where the Tamm mode is hybridized with two neighboring microcavity modes simultaneously. The possibility of tuning the hybrid mode wavelengths by selecting a thickness of the first photonic crystal layer adjacent to the silver layer was demonstrated.

The possibility of temperature and electric-field tuning of the hybrid mode wavelengths and light energy localization at the PC surface is important for TPP applications in sensing, lasing, solar photovoltaics, WOLED technology, and nonlinear control.

\section{ACKNOWLEDGMENTS}

The reported study was funded by Russian Foundation for Basic Research, Government of Krasnoyarsk Territory, Krasnoyarsk Region Science and Technology Support Fund to the research project no. 17-42-240464. P.S.P. acknowledges the support of the Scholarship of the President of the Russian Feder- ation no. SP-227.2016.5. The authors thank D.N. Gulkin and Dr. V.O. Bessonov for help and fruitful discussions.

\section{REFERENCES}

1. A. Kavokin, I. Shelykh, and G. Malpuech, "Optical Tamm states for the fabrication of polariton lasers," Appl. Phys. Lett. 87, 261105 (2005).

2. A. V. Kavokin, I. A. Shelykh, and G. Malpuech, "Lossless interface modes at the boundary between two periodic dielectric structures," Phys. Rev. B 72, 1-4 (2005).

3. I. V. Timofeev and S. Y. Vetrov, "Chiral optical Tamm states at the boundary of the medium with helical symmetry of the dielectric tensor," JETP Lett. 104, 380-383 (2016).

4. A. Namdar, I. V. Shadrivov, and Y. S. Kivshar, "Backward Tamm states in left-handed metamaterials," Applied Physics Letters 89, 114104 (2006).

5. M. Kaliteevski, I. Iorsh, S. Brand, R. A. Abram, J. M. Chamberlain, A. V. Kavokin, and I. A. Shelykh, "Tamm plasmon-polaritons: Possible electromagnetic states at the interface of a metal and a dielectric Bragg mirror," Phys. Rev. B 76, 165415 (2007).

6. M. E. Sasin, R. P. Seisyan, M. A. Kalitteevski, S. Brand, R. A. Abram, J. M. Chamberlain, A. Y. Egorov, A. P. Vasil'ev, V. S. Mikhrin, and A. V. Kavokin, "Tamm plasmon polaritons: Slow and spatially compact light," Appl. Phys. Lett. 92, 251112 (2008).

7. S. Y. Vetrov, R. G. Bikbaev, and I. V. Timofeev, "Optical Tamm states at the interface between a photonic crystal and a nanocomposite with resonance dispersion," J. Exp. Theor. Phys. 117, 988-998 (2013).

8. C. Symonds, A. Lemaître, P. Senellart, M. H. Jomaa, S. Aberra Guebrou, E. Homeyer, G. Brucoli, and J. Bellessa, "Lasing in a hybrid GaAs/silver Tamm structure," Applied Physics Letters 100, 121122 (2012).

9. A. R. Gubaydullin, C. Symonds, J. Bellessa, K. A. Ivanov, E. D. Kolykhalova, M. E. Sasin, A. Lemaitre, P. Senellart, G. Pozina, and M. A. Kaliteevski, "Enhancement of spontaneous emission in Tamm plasmon structures," Scientific Reports 7, 9014 (2017).

10. O. Gazzano, S. Michaelis de Vasconcellos, K. Gauthron, C. Symonds, P. Voisin, J. Bellessa, A. Lemaître, and P. Senellart, "Single photon source using confined Tamm plasmon modes," Applied Physics Letters 100, 232111 (2012).

11. B. I. Afinogenov, A. A. Popkova, V. O. Bessonov, and A. A. Fedyanin, "Measurements of the femtosecond relaxation dynamics of Tamm plasmon-polaritons," Applied Physics Letters 109, 171107 (2016).

12. W. L. Zhang and S. F. Yu, "Bistable switching using an optical Tamm cavity with a Kerr medium," Opt. Commun. 283, 2622-2626 (2010).

13. S. Y. Vetrov, P. S. Pankin, and I. V. Timofeev, "The optical Tamm states at the interface between a photonic crystal and a nanocomposite containing core-shell particles," J. Opt. (United Kingdom) 18, 65106 (2016).

14. Z.-Y. Yang, S. Ishii, T. Yokoyama, T. D. Dao, M.-g. Sun, T. Nagao, and K. P. Chen, "Tamm plasmon selective thermal emitters," Opt. Lett. 41, 4453 (2016).

15. Z.-Y. Yang, S. Ishii, T. Yokoyama, T. D. Dao, M.-G. Sun, P. S. Pankin, I. V. Timofeev, T. Nagao, and K.-P. Chen, "Narrowband Wavelength Selective Thermal Emitters by Confined Tamm Plasmon Polaritons," ACS Photonics 4, 2212-2219 (2017).

16. A. P. Vinogradov, A. V. Dorofeenko, S. G. Erokhin, M. Inoue, A. A. Lisyansky, A. M. Merzlikin, and A. B. Granovsky, "Surface state peculiarities in one-dimensional photonic crystal interfaces," Phys. Rev. B 74, 1-8 (2006).

17. C. Symonds, A. Lemaître, E. Homeyer, J. C. Plenet, and J. Bellessa, "Emission of Tamm plasmon/exciton polaritons," Applied Physics Letters 95, 151114 (2009).

18. M. Kaliteevski, S. Brand, R. A. Abram, I. Iorsh, A. V. Kavokin, T. C. H. Liew, and I. A. Shelykh, "Hybrid states of Tamm plasmons and excitonpolaritons," Superlattices Microstruct. 49, 229-232 (2011).

19. S. S.-U. Rahman, T. Klein, S. Klembt, J. Gutowski, D. Hommel, and K. Sebald, "Observation of a hybrid state of Tamm plasmons and microcavity exciton polaritons," Scientific Reports 6, 34392 (2016).

20. A. V. Baryshev, K. Kawasaki, P. B. Lim, and M. Inoue, "Interplay of surface resonances in one-dimensional plasmonic magnetophotonic crystal slabs," Physical Review B 85, 205130 (2012).

21. H. Liu, X. Sun, F. Yao, Y. Pei, F. Huang, H. Yuan, and Y. Jiang, "Optical 
magnetic field enhancement through coupling magnetic plasmons to Tamm plasmons." Opt. Express 20, 19160-19167 (2012).

22. B. I. Afinogenov, V. O. Bessonov, A. A. Nikulin, and A. A. Fedyanin, "Observation of hybrid state of Tamm and surface plasmon-polaritons in one-dimensional photonic crystals," Appl. Phys. Lett. 103, 61112 (2013).

23. I. Iorsh, P. V. Panicheva, I. A. Slovinskii, and M. A. Kaliteevski, "Coupled Tamm plasmons," Technical Physics Letters 38, 351-353 (2012).

24. S. Y. Vetrov, P. S. Pankin, and I. V. Timofeev, "Coupled Optical Tamm States at Edges of a Photonic Crystal Enclosed by a Composite of Core-Shell Nanoparticles," Physics of Wave Phenomena 25, 170-174 (2017).

25. M. Kaliteevski, S. Brand, R. Abram, I. Iorsh, A. Kavokin, T. Liew, and I. Shelykh, "Hybrid states of Tamm plasmons and exciton-polaritons," Superlattices and Microstructures 49, 229-232 (2011).

26. R. Brückner, M. Sudzius, S. I. Hintschich, H. Fröb, V. G. Lyssenko, and K. Leo, "Hybrid optical Tamm states in a planar dielectric microcavity," Physical Review B 83, 033405 (2011).

27. R. Brückner, M. Sudzius, S. I. Hintschich, H. Fröb, V. G. Lyssenko, M. A Kaliteevski, I. Iorsh, R. A. Abram, A. V. Kavokin, and K. Leo, "Parabolic polarization splitting of Tamm states in a metal-organic microcavity," Applied Physics Letters 100, 062101 (2012).

28. X.-L. Zhang, J.-F. Song, J. Feng, and H.-B. Sun, "Spectral engineering by flexible tunings of optical Tamm states and Fabry-Perot cavity resonance," Optics Letters 38, 4382 (2013).

29. X.-L. Zhang, J.-F. Song, X.-B. Li, J. Feng, and H.-B. Sun, "Light trapping schemes in organic solar cells: A comparison between optical Tamm states and Fabry-Pérot cavity modes," Organic Electronics 14, 15771585 (2013).

30. X.-L. Zhang, J. Feng, X.-C. Han, Y.-F. Liu, Q.-D. Chen, J.-F. Song, and H.-B. Sun, "Hybrid Tamm plasmon-polariton/microcavity modes for white top-emitting organic light-emitting devices," Optica 2, 579 (2015).

31. Y.-t. Fang, L.-x. Yang, W. Kong, and N. Zhu, "Tunable coupled states of a pair of Tamm plasmon polaritons and a microcavity mode," Journal of Optics 15, 125703 (2013).

32. M. A. Kaliteevski, A. A. Lazarenko, N. D. Il'inskaya, Y. M. Zadiranov, M. E. Sasin, D. Zaitsev, V. A. Mazlin, P. N. Brunkov, S. I. Pavlov, and A. Y. Egorov, "Experimental Demonstration of Reduced Light Absorption by Intracavity Metallic Layers in Tamm Plasmon-based Microcavity," Plasmonics 10, 281-284 (2015).

33. H.-X. Da, Z.-Q. Huang, and Z. Y. Li, "Electrically controlled optical Tamm states in magnetophotonic crystal based on nematic liquid crystals," Optics Letters 34, 1693 (2009).

34. J. Luo, P. Xu, and L. Gao, "Controllable switching behavior of optical Tamm state based on nematic liquid crystal," Solid State Communications 151, 993-995 (2011).

35. C. Y. Chang, Y. H. Chen, Y. L. Tsai, H. C. Kuo, and K. P. Chen, "Tunability and Optimization of Coupling Efficiency in Tamm Plasmon Modes," IEEE J. Sel. Top. Quantum Electron. 21, 262-267 (2015).

36. V. G. Arkhipkin, V. A. Gunyakov, S. A. Myslivets, V. P. Gerasimov, V. Y. Zyryanov, S. Y. Vetrov, and V. F. Shabanov, "One-dimensional photonic crystals with a planar oriented nematic layer: Temperature and angular dependence of the spectra of defect modes," J. Exp. Theor. Phys. 106, 388-398 (2008).

37. V. Y. Zyryanov, S. A. Myslivets, V. A. Gunyakov, A. M. Parshin, V. G. Arkhipkin, V. F. Shabanov, and W. Lee, "Magnetic-field tunable defect modes in a photonic-crystal/liquid-crystal cell." Opt. Express 18, 12831288 (2010).

38. V. G. Arkhipkin, V. A. Gunyakov, S. A. Myslivets, V. Y. Zyryanov, V. F. Shabanov, and W. Lee, "Electro- and magneto-optical switching of defect modes in one- dimensional photonic crystals," J. Exp. Theor. Phys. 112, 577-587 (2011).

39. P. B. Johnson and R. W. Christy, "Optical Constants of the Noble Metals," Phys. Rev. B 6, 4370-4379 (1972).

40. P. Yeh, "Electromagnetic propagation in birefringent layered media," J. Opt. Soc. Am. 69, 742-756 (1979).

41. H. J. Deuling, "Deformation of Nematic Liquid Crystals in an Electric Field," Mol. Cryst. Liq. Cryst. 19, 123-131 (1972).

42. J. Li, C.-H. Wen, S. Gauza, R. Lu, and S.-T. Wu, "Refractive Indices of Liquid Crystals for Display Applications,” J. Disp. Technol. 1, 51-61 (2005).

43. I. V. Timofeev, Y. T. Lin, V. A. Gunyakov, S. A. Myslivets, V. G. Arkhipkin, S. Y. Vetrov, W. Lee, and V. Y. Zyryanov, "Voltage-induced defect mode coupling in a one-dimensional photonic crystal with a twisted-nematic defect layer," Phys. Rev. E 85, 011705(7) (2012).

44. M. S. Sefton, A. R. Bowdler, and H. J. Coles, "Light Scattering Studies of the Viscoelastic Ratios of Mixtures of Side Chain Liquid Crystalline Polymers in Low Molar Mass Mesogens," Molecular Crystals and Liquid Crystals 129, 1-16 (1985).

45. M. Born and E. Wolf, Principles of Optics: Electromagnetic Theory of Propagation, Interference and Diffraction of Light (Cambridge University Press, 1999).

46. L. M. Blinov, Structure and Properties of Liquid Crystals, Topics in applied physics (Springer, 2010).

47. G. A. Korn and T. M. Korn, Mathematical Handbook for Scientists and Engineers: Definitions, Theorems, and Formulas for Reference and Review (Dover Civil and Mechanical Engineering) (Dover Publications, 2000), 2nd ed.

48. E. Nowinowski-Kruszelnicki, J. Kȩdzierski, Z. Raszewski, L. Jaroszewicz, M. Kojdecki, W. Piecek, P. Perkowski, M. Olifierczuk, E. Miszczyk, K. Ogrodnik, and P. Morawiak, "Measurement of elastic constants of nematic liquid crystals with use of hybrid in-plane-switched cell," Opto-Electronics Review 20, 255-259 (2012).

49. H. A. Haus, Waves and fields in optoelectronics, Prentice-Hall Series in Solid State Physical Electronics (Prentice Hall, Incorporated, Upper Saddle River, NJ, USA, 1983).

50. M. I. Rabinovich and D. I. Trubetskov, Oscillations and Waves: in Linear and Nonlinear Systems (Mathematics and its Applications) (Springer, 1989), 1989th ed

51. F. Peng, Y. Huang, F. Gou, M. Hu, J. Li, Z. An, and S.-T. Wu, "High performance liquid crystals for vehicle displays," Optical Materials Express 6, 717 (2016). 